\title{
PLACE THE CONTROLLERS WHEREVER YOU WANT: USABILITY AND USER EXPERIENCE OF CUSTOMISABLE MUSICAL DEVICES
}

\author{
Matteo Fanchin ${ }^{1}$, Patrik Pluchino ${ }^{2}$ and Luciano Gamberini ${ }^{2}$ \\ ${ }^{1}$ Department of General Psychology \\ ${ }^{2}$ Department of General Psychology, Human Inspired Technology (HIT) Research Centre \\ Via Venezia 8, Padova, 35131, Italy
}

\begin{abstract}
The current study investigates how different types of multi-effects guitar devices can affect users' evaluations of usability and user experience. Participants were asked to interact with three different multi-effects devices while playing an electric guitar. Typically multi-effect pedalboards are used by musicians to modify an instrument sound. These tools allow us to distort notes or to change the duration of the produced sounds. The main difference between the three devices pertains to the placement of their controllers: fixed-pedals on the ground; movable-pedals on the ground; movable and wireless pedals that can be freely placed, at the discretion of the user (e.g., on the guitar, the user's body). The second and third devices were both prototypes designed and developed by the first author. Several usability dimensions (e.g., ease of use, enjoyment, etc.) were evaluated (i.e., ad hoc questionnaire). Besides the musical experience also the attitude towards novel technologies (i.e., personal innovativeness) was collected. Overall, the two prototypes appeared as easier to use and enjoyable compared to the fixed-pedals device. Furthermore, participants with high levels of personal innovativeness perceived the devices as more controllable. The opportunity of customizing the placement of the pedals positively affected the evaluations. The study outcomes lay the foundations for the implementation of new prototypes that could be tailored based on musicians' needs. The result will be an increment in usability and a more positive user experience.
\end{abstract}

\section{KEYWORDS}

Prototyping, Human-Computer Interaction, Usability, User Experience

\section{INTRODUCTION}

Nowadays, new technologies are changing not only the way we interact with musical instruments, and their hardware-related devices, but also the actual physical features of the instruments themselves (Saffer, 2008; Moggridge and Atkinson, 2007; Medeiros et al., 2014; Holland et al., 2013, 2016; Tanaka, 2019). Contemporary musical instruments are increasingly digitized and they become closely linked to computers (Cook, 2017). Effects units, music software, digital audio workstations, pre-amplifiers are only a few of the tools that musicians have to learn and deeply understand, in terms of their functioning, both in a solo performance or while they are playing in a band (Wessel and Wright, 2002; Paine, 2013). Furthermore, a recent paper highlighted the vision and challenges related to the Internet of Musical Things (i.e., IoMusT; Turchet et al., 2018). This domain considers, for instance, smart instruments, wireless and wired networks, and service for controlling parameters of Musical Things. The aim is enriching the overall experience of concerts (i.e., augmented and immersive), increasing audience involvement, improving e-learning of music, and the quality of music studio production (Turchet et al., 2018; Turchet, 2018). This new perspective goes in the direction of design (Morreale, De Angeli, and O'Modhrain, 2014) and developing products that could be easier to use, accepted, and more adaptable to the users' needs. In such circumstances, the musicians will consider these devices as tools that could enhance their performance skills, and as a consequence, their satisfaction within the performance. An additional result would be a higher intention to use. Fernandes and Holmes (Fernandes and Holmes, 2002) underlined how a musical device that does not match the above-mentioned requirements hinders the user's creative process by increasing cognitive demands. In contrast, user interfaces that are intuitive and clear, minimize interruptions to the spontaneity of performance or composition. According to Wanderley and Orio (2002), HCI methodology could be a suitable tool to evaluate the perceived usability and user experience of musical interfaces. 


\subsection{Usability and User Experience}

When designing interactive systems, one of the main goals is that they should be usable for the users. The usability evaluation has to take into account the context of the application, the end-users, and the characteristics of the systems themselves (Sharp, Preece, and Rogers, 2019). Usability is a multidimensional construct that permits to investigate different aspects of the interaction with a tool (e.g., ease of use, safety, learnability). In literature, there are plenty of definitions but the most authoritative is the one provided by the International Organization for Standardization (ISO, 2009): "The effectiveness, efficiency and satisfaction with which specified users achieve specified goals in particular environments" (Boehm, Brown, and Lipow, 1976). In the present context, the effectiveness is considered as the accuracy and completeness with which users can achieve specific goals in particular environments. The efficiency refers to the resources invested concerning the accuracy and completeness of goals achieved. The comfort and acceptability of the working system might be affected also by its actual usage (Bevan, 2006). Higher usability increases perceived efficiency of a system (Sharp, Preece, and Rogers, 2019). Furthermore, usable systems positively affect the safety of the HCI interaction, decreasing the chance of errors, reducing the time of training, and the support needed by the users. The usability has also a relevant impact on the success of a product on the market: when two systems present the same functionality and similar prices the users will choose the one that better supports the interaction. While in the assessment of usability the primary focus is on the performance and fluid interaction, the user experience (UX) takes in consideration desirable (e.g., satisfaction, engagement, enjoyment) and undesirable (e.g., unpleasantness, frustration, boredom) aspects of the interaction (Sharp, Preece, and Rogers, 2019). The term "User Experience" was coined to describe what a person feels when interacting or imagining to interact with a prototype, a final product, a system, or a service. The International Organization of Standardization defined UX as: "a person's perceptions and responses that result from the use or anticipated use of a product, system or service" ISO (2009). As already mentioned, the previous experiences of use are part of the UX and they are relevant to consider when the designer aims to develop a new product or to improve a product that is already on the market. The various dimensions of UX can be evaluated during the entire lifecycle of a product (i.e. early prototypes, launch into the market, and product abandonment).

\section{METHOD}

\subsection{Participants}

Forty-two students from University of XX took part in the main study $(\mathrm{F}=2, \mathrm{M}=40$, mean age $=27.73$, $\mathrm{SD}=2.36$ ). Twenty-one participants belonged to the category of experienced guitar players (i.e., nine years of active study of guitar and/or graduated at the conservatory). Besides, they were frequent users of multi-effects pedalboards. Twenty-one participants were considered beginners guitar players. Among all the participants, 21 usually use a pedalboard, 13 rarely, and 8 have never used it. A total of 11 participants have positioned the second prototype (wireless) on the ground, 13 on the guitar, and 18 chose mixed positions (i.e., ground, guitar, and/or body parts; see 3.2.3).

\subsection{Experimental Design}

Two factors were independently manipulated within the current study ( $3 \mathrm{X} 2$ mixed-design). The within-participants factor was the type of multi-effect device (three levels: fixed-pedals on the ground vs. movable-pedals on the ground vs. movable and wireless pedals; see Figures 1, 2, 3) utilized in the three musical sessions. The between-participants factor was the musical experience of participants (two levels: beginners vs. experts). The order of presentation of the multi-effect units was counterbalanced across participants. The between-participants factor was the musical experience of participants (two levels: beginners vs. experts). Following the literature, we defined the cut-off for considering an expert musician as an active study of an instrument for at least nine years or the attendance/graduation from a conservatory (Gaser and Schlaug, 2003; Hanna-Pladdy and MacKay, 2011), and the frequent use of multi-effect pedalboards. 


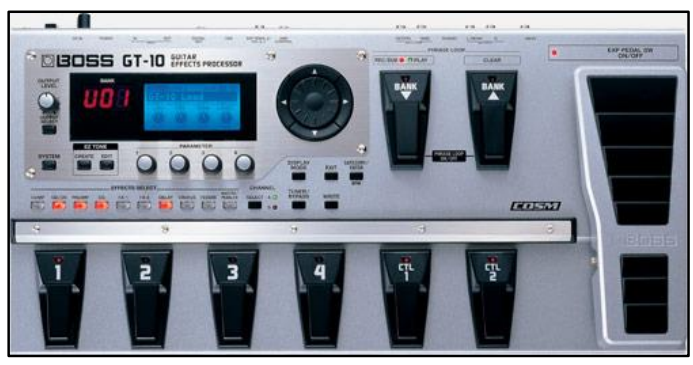

Figure 1. Traditional Pedalboard (BOSS GT-10; i.e., fixed-pedals on the ground)

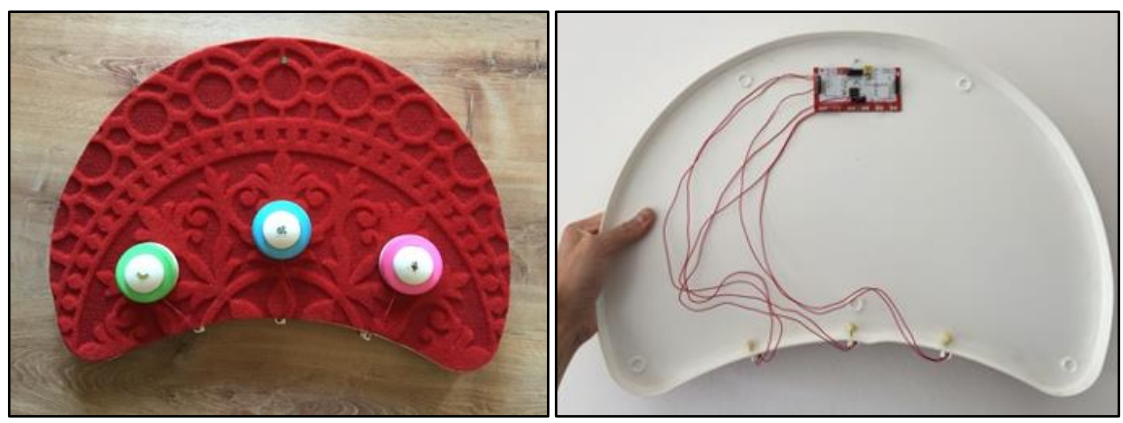

Figure 2. First Prototype (MakeyMakey based; i.e, movable-pedals on the ground)

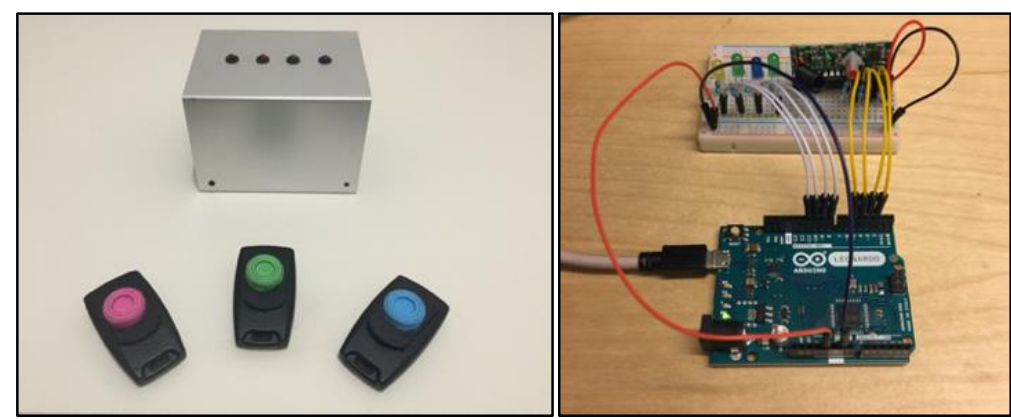

Figure 3. Second Prototype (Arduino Leonardo Based; i.e., movable and wireless pedals)

\subsection{Experimental Equipment and Materials}

The following equipment and materials were utilized in the study.

An MSI Notebook GE72 6QD was used to run the software Albleton 9 Live and to power the M-Audio Fast Track Pro Audio/MIDI interface. Participants wore a pair of Sony MDR-XB200 headphones to listen to the sounds output. A Samsung Notebook NP300E5C was employed to allow participants to fill out the questionnaires (using Google Modules).

BOSS GT-10 (i.e., fixed-pedals device). A traditional pedalboard already on the market (see Figure 1).

First prototype (i.e., movable-pedals device). A pedalboard realized utilizing the MakeyMakey board with wired but movable controllers (see Figure 2). Participants were allowed to customize the positions of the controllers on the external surface (i.e., red in Figure 2) of the device. The controllers were attached with Velcro on the chosen positions of the device surface.

Second prototype. A pedalboard realized utilizing the Arduino Leonardo microcontroller board (i.e., movable and wireless pedals device). The device comprises four parts: the central box containing the Arduino, three completely wireless controllers. The absence of wires allowed participants to freely choose where to place the three controllers. The controllers were attached with Velcro on the chosen positions.

Pre-Test questionnaire. This tool contained different items regarding general information 
(e.g. demographic, musical experience) and three items that evaluated the Personal Innovativeness of users. The gathered data allowed us to divide the participants' sample into two groups: beginners and expert musicians. Instead, the Personal Innovativeness dimension was considered to subsequently investigate if the judgments given to the items presented in the post-test questionnaires were influenced by participants' attitude towards the novel information technology (Agarwal and Prasad, 1998).

Post-test questionnaire. The second tool was administered to collect information about the participants' perceived usability and user experience of the various multi-effect devices. The same questionnaire was filled out three times during the experiment, namely after each session (Boehm, Brown, and Lipow, 1976). The presentation of the items was randomized.

\subsection{Procedure}

Upon arrival, at one of the laboratories of the Department XX of the University of XX, participants were welcomed and asked to read carefully a document containing general information about the study. Then, they were administered with an informed consent. Afterward, participants filled out the Pre-Test questionnaire. Based on a literature review (Barbosa et al., 2011), participants performed three sessions of practice to familiarize with the controllers of each multi-effect device respectively before each of the three performance sessions (i.e., one for each pedalboard; see Figure 4 for details). They were asked to play a song at leisure with the guitar and to interact at least once with each of the three controllers available on the multi-effects devices. Note that the fixed-pedals device presents six controllers but only four could be used. Concerning the two prototypes, participants had an additional temporal interval that allowed the decision of where to place the controllers. For the whole duration of the experiment, the researcher remained inside the experimental room, although out of participants' sight. A complete experiment lasted about one hour.

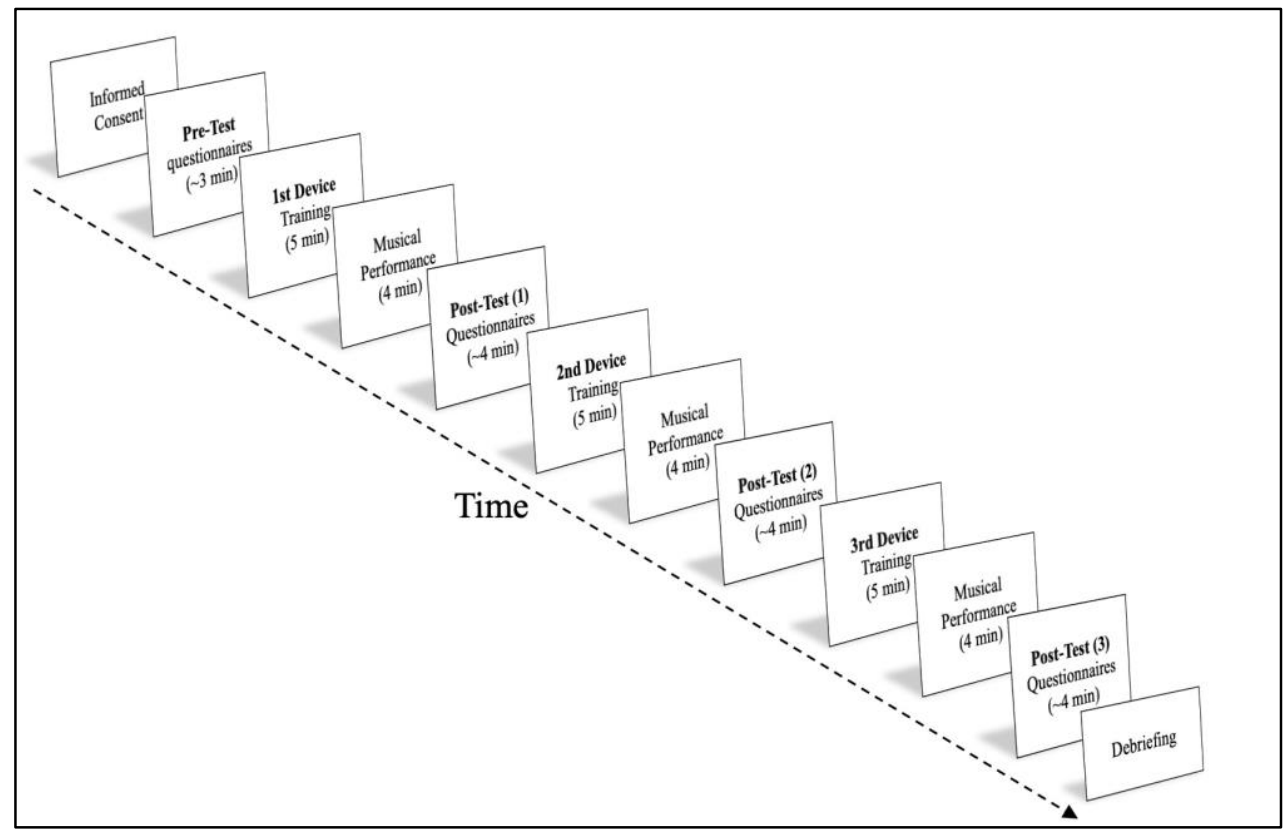

Figure 4. Experimental Procedure

\section{DATA ANALYSIS AND RESULTS}

All the statistical analyses were performed utilizing the R Studio software (Core Team, 2019). Non-parametric tests were used in so far as the considered dependent variables were ordinals (i.e. Likert scale scores). 


\subsection{Pre-Test (Personal Innovativeness and Expertise Level)}

A series of Mann-Whitney $U$ tests was performed to analyze potential differences in the participants' attitude towards information technology due to their musical experience. No main effects due to the musical experience emerged $(p>.05)$. The beginners and experts showed similar mean scores to the personal innovativeness construct.

\subsection{Post-Test}

\subsubsection{Usability and UX Items and Device}

A series of Friedman tests was performed in order to analyze potential differences due to the three different multi/effect devices. An effect emerged considering each of the following dimensions: Perceived Ease of Use $\left(\mathrm{PEOU} ; X^{2}(2)=12.24, p<.01\right)$, Perceived External Control $\left(\mathrm{PEC} ; X^{2}(2)=18.85, p<.001\right)$, Enjoyment (ENJ; $\left.X^{2}(2)=30.75, p<.001\right)$, Sound Output Quality (SOUT; $\left.X^{2}(2)=20.28, p<.001\right)$, Interface Output Quality $\left(\right.$ IOUT; $\left.X^{2}(2)=12.25, p<.001\right)$, Lights Out-put Quality $\left(\right.$ LOUT; $\left.X^{2}(2)=9.72, p<.001\right)$, and Intent of Use (INT; $\left.X^{2}(2)=36.20, p<.001\right)$. Pairwise comparisons using Wilcoxon tests were performed to compare the median scores given to the different devices (see Tab. 1,2). The $p$-values were adjusted using the Bonferroni correction.

Table 1. $p$-values (Usability and UX items) as a function of the device

\begin{tabular}{llll}
\hline Constructs & $1 \mathrm{Vs} 2$ & $1 \mathrm{Vs} 3$ & $2 \mathrm{Vs} 3$ \\
\hline PEOU & $p<.05^{*}$ & $p<.001^{* * *}$ & $p>.05$ \\
PEC & $p<.05^{*}$ & $p<.01^{* *}$ & $p>.05$ \\
ENJ & $p<.001^{* * *}$ & $p<.01^{* *}$ & $p>.05$ \\
SOUT & $p<.05^{*}$ & $p<.05^{*}$ & $p>.05$ \\
IOUT & $p>.05$ & $p<.05^{*}$ & $p>.05$ \\
LOUT & $p<.001^{* * *}$ & $p>.05$ & $p<.01^{* *}$ \\
INT & $p<.01^{* *}$ & $p<.001^{* * *}$ & $p>.05$ \\
\hline
\end{tabular}

Table 2. Median scores (Usability and UX items) as a function of the device

\begin{tabular}{llll}
\hline Constructs & Traditional & Prototype 1 & Prototype 2 \\
\hline PEOU & $M d n=4.75$ & $M d n=5.50$ & $M d n=6$ \\
PEC & $M d n=5.33$ & $M d n=5.83$ & $M d n=6$ \\
ENJ & $M d n=5$ & $M d n=5.50$ & $M d n=6.33$ \\
SOUT & $M d n=4.33$ & $M d n=5.66$ & $M d n=5.66$ \\
IOUT & $M d n=4.50$ & $M d n=5.16$ & $M d n=5.50$ \\
LOUT & $M d n=4.66$ & $M d n=5.33$ & $M d n=4.66$ \\
INT & $M d n=3$ & $M d n=4.66$ & $M d n=5.33$ \\
\hline
\end{tabular}

\subsubsection{Usability and UX Items and Expertise Level}

A series of Mann-Whitney $U$ tests was carried out to evaluate the perceived usability and UX items (PEOU, PEC, ENJ, SOUT, IOUT, LOUT, INT) as a function of the expertise level. A difference emerged considering respectively the Perceived Enjoyment $(W=2502.5, p<.05)$ and the Sound Output Quality $(W=2614, p<.01)$. These $p$-values were adjusted for multiple comparisons utilizing the BH method (Benjamini \& Hochberg, 1995).

\subsubsection{Usability and UX Items, Devices and Placement}

A series of Friedman tests was conducted to evaluate the perceived usability and the user experience as a function of the placement of the controllers. Three groups of participants were identified in terms of where they placed the movable and wireless pedals (i.e., on the ground, on the guitar, or in a mixed position). 
Significant differences emerged only in the group of mixed positions (i.e., movable and wireless pedals) compared to the first prototype (i.e., movable pedals) and the traditional pedalboard (i.e., fixed-pedals). In particular, the differences were shown with the PEOU $\left(X^{2}(2)=9.48, p<.01\right.$; see Figure 5), ENJ $\left(X^{2}(2)=18\right.$, $p<.001$; see Figure 6$)$, SOUT $\left(X^{2}(2)=18.5, p<.001\right.$; see Figure 7) and INT $\left(X^{2}(2)=23.85, p<.001\right.$, see Figure 8) dimensions. The subsequent Wilcoxon tests showed that the PEOU scores were higher for the second prototype compared to the traditional device $(p<.05)$. The ENJ scores were higher for both the first $(p<.01)$ and the second prototype $(p<.001)$ in comparison to the traditional device. Higher scores in terms of SOUT were assigned to both the first $(p<.001)$ and the second prototype $(p<.05)$ compared to the ones of the traditional device. Finally, considering the INT dimension, higher scores were associated with both the first $(p<.05)$ and the second prototype $(p<.05)$ compared to the ones of the traditional device.

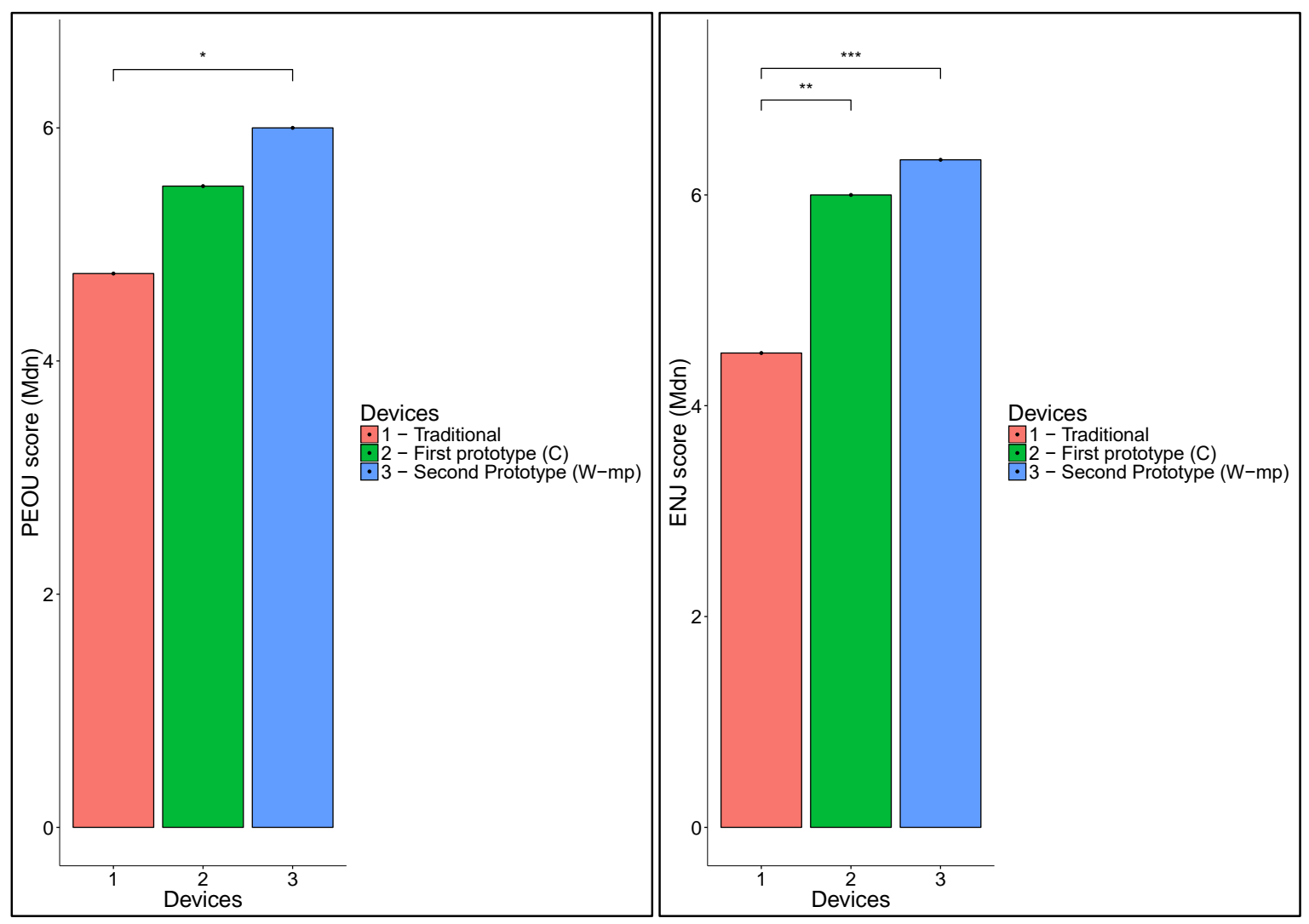

Figure 5 and 6.5 (left) Median scores of Perceived Ease of Use as a function of devices in the mixed position group. $\mathrm{C}=$ customized; $\mathrm{W}-\mathrm{mp}=$ Wireless mixed-position. 6 (right) Median scores of Perceived Enjoyment as a function of devices in the mixed position group. $\mathrm{C}=$ customized; $\mathrm{W}$-mp $=$ Wireless mixed-position 


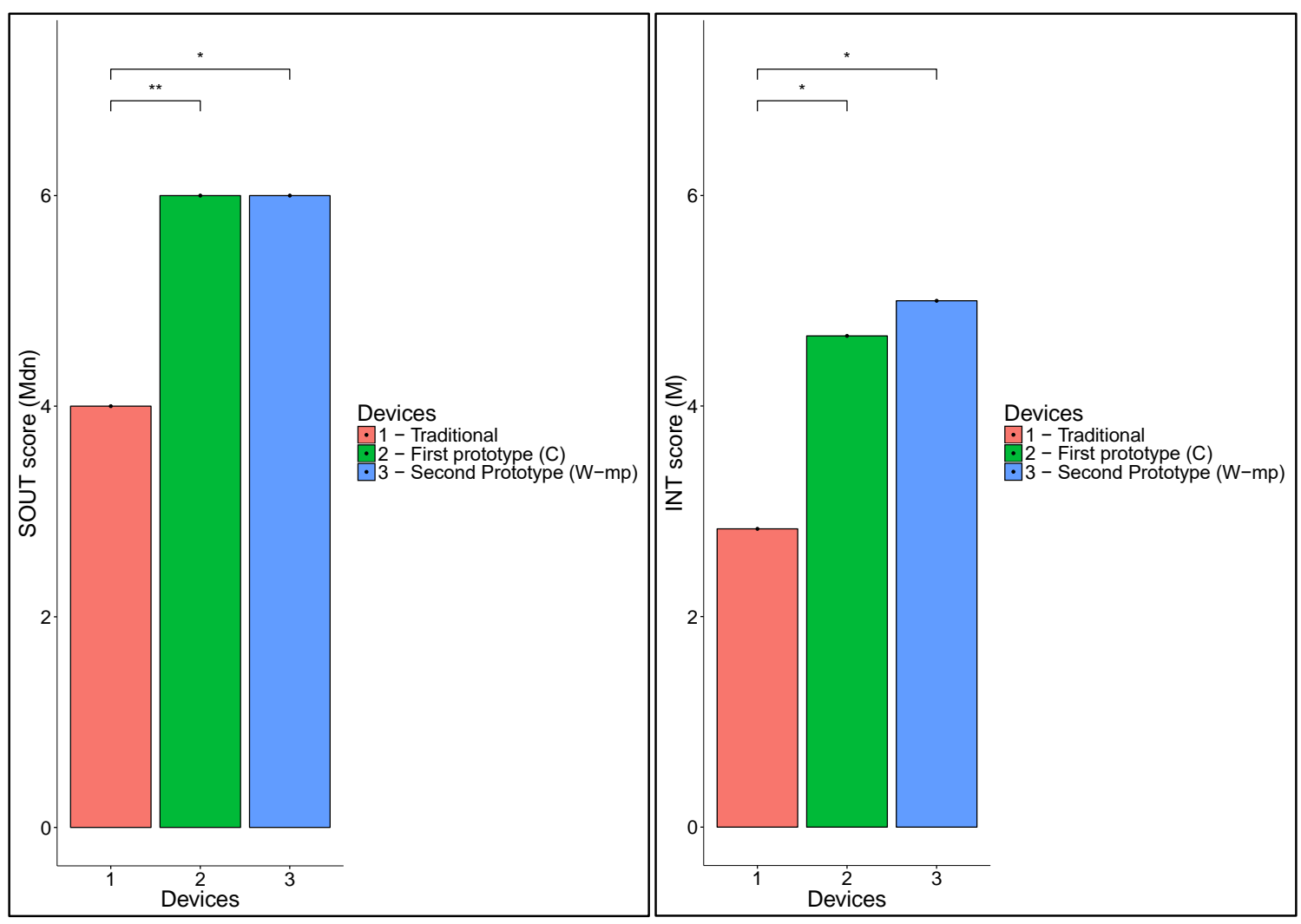

Figure 7 and 8.7 (left) Median scores of Sound Output Quality as a function of devices in the mixed position group. $\mathrm{C}=$ customized; $\mathrm{W}-\mathrm{mp}=$ Wireless mixed-position. 8 (right) Median scores of Intent to Use as a function of devices in the mixed position group. $\mathrm{C}=$ customized; $\mathrm{W}-\mathrm{mp}=$ Wireless mixed-position

\subsubsection{Usability and UX items and Personal Innovativeness}

To investigate whether the attitude towards information technology could affect the usability and UX, a median split method was utilized to obtain two different groups of participants: high vs. low level of personal innovativeness (i.e., $M d n=4)$. A series of Wilcoxon tests was performed and a significant effect emerged considering only with the Perceived Of External Control construct $(W=2341, p<.001)$.

\section{CONCLUSION}

The present study aimed to investigate how the features of three different multi-effects pedalboards for guitar affected their evaluation in terms of usability and UX. Furthermore, also the influence of musical experience (i.e. beginners vs. experts) and Personal Innovativeness were considered. Concerning to the usability and user experience as a function of the device, overall higher scores were assigned to the first (i.e., movable pedals) and the second prototype (i.e., movable and wireless pedals) compared to the traditional pedalboard (i.e., fixed-pedals). Findings showed that both prototypes appeared as more ease of use, more controllable, and enjoyable compared to the traditional device. Considering the quality of visual feedback a difference emerged. The second prototype received a better evaluation compared to the first one. This could be due to the presence, in the second prototype, of led lights with different colors (i.e., dedicated to each sound-effect). This feature helped the musician to promptly and effectively recognize which effect he/she had currently selected. Furthermore, an effect emerged considering the expertise level of participants. Both beginners and experts evaluated the prototypes as more enjoyable compared to the traditional device (i.e., fixed-pedals). The expertise level highlighted another interesting outcome. The beginner's group only evaluated the Sound Output Quality (SOUT) better while considering the second prototype (i.e., movable and wireless pedals) in comparison with the traditional pedalboard. An explanation can be that the experts are familiar with the small distortions in the 
sounds that characterize an analogic pedalboard (i.e., the traditional one) in contrast to the clean sound that is produced by digital pedalboards (i.e., first and second prototypes). Thus, experts seemed to be able to recognize that the variation in sound quality was due to a different physical architecture of the pedalboard (i.e., analogic vs. digital) and not to the quality of the sound output itself. Considering the second prototype as the most advanced one, due to its customizable architecture, the authors deeply investigate the effect of the placement of the wireless pedals (i.e., on the ground, on the guitar, or in mixed positions, namely on the guitar, ground and/or musician's body). The emerged differences supported the assumption that the opportunity to explore a novel mixed and free pedals placement positively affected the perceived usability and UX. Besides, the attitude towards information technology was affecting usability and user experience. Indeed, a difference emerged comparing the usability and UX dimensions as a function of the two Personal Innovativeness groups (High vs. Low). A higher predisposition to information technology resulted in higher scores on the Perceived of External Control (PEC) construct. This result suggests that people who consider themselves to be more attracted by novel technologies perceived the presented prototypes more controllable. This research study underlined that there are no limitations to where a musician can place the controllers of multi-effect devices. For instance, participants of mixed-positions found new ways of interaction as a result of the freedom in the placement of the controllers (e.g., behind the neck of the guitar, between the knees and under the armpits). These were not foreseen before the study. Furthermore, the possibility of a high level of personalization led to more positive evaluation, in terms of usability and completely new interaction experience with musical devices, compared to a situation in which the multi-effect devices cannot be customized (i.e., traditional pedalboard with

fixed-pedals). In general, making devices more usable for novices is crucial to reduce the traditional barriers that may hamper the initial phases of learning how to play a musical instrument (McPherson, Morreale, and Harrison, 2019). However, some limitations of the study have to be pointed out. It should be crucial to increase the numerosity of the sample in the investigation of the wireless prototype especially regarding the positions chosen by participants for the pedals placement. A second limitation pertains to the sample composition (gender), herein the females involved were only two in the face of forty males, which limits the generalizability of the results. Finally, a potential development should be the further customization of the prototypes adding new pedals of different sizes and/or that provide different functions.

\section{ACKNOWLEDGEMENT}

The study was supported by a grant from MIUR (Dipartimenti di Eccellenza DM 11/05/2017 n. 262) to the Department of General Psychology.

\section{REFERENCES}

Arduino Leonardo, https: //www.arduino.cc/en/Main/ArduinoBoardLeonardo

BOSS GT-10, https://www.boss.info/us/products/gt-10/

Agarwal, R. and Prasad, J., 1998. A conceptual and operational definition of personal innovativeness in the domain of information technology. Information systems research, 9(2), pp.204-215.

Barbosa, J., Calegario, F., Magalhães, F., Teichrieb, V., Ramalho, G. and Cabral, G., 2011, January. Towards an evaluation methodology for digital music instruments considering performer's view: a case study. In Proceedings of 13th Brazilian Symposium on Computer Music.

Benjamini, Y. and Hochberg, Y., 1995. Controlling the false discovery rate: a practical and powerful approach to multiple testing. Journal of the Royal statistical society: series B (Methodological), 57(1), pp.289-300.

Bevan, N., 2006. International standards for HCI. In Encyclopedia of human computer interaction (pp. 362-372). IGI Global.

Boehm, B.W., Brown, J.R. and Lipow, M., 1976, October. Quantitative evaluation of software quality. In Proceedings of the 2nd international conference on Software engineering (pp. 592-605). IEEE Computer Society Press.

Cook, P., 2017. 2001: Principles for Designing Computer Music Controllers. In A NIME Reader (pp. 1-13). Springer, Cham. 
Faulkner, L., 2003. Beyond the five-user assumption: Benefits of increased sample sizes in usability testing. Behavior Research Methods, Instruments, \& Computers, 35(3), pp.379-383.

Fernandes, G. and Holmes, C., 2002, April. Applying HCI to music-related hardware. In CHI'O2 Extended Abstracts on Human Factors in Computing Systems (pp. 870-871).

ISO, International Organization for Standardization, http://www.iso.org

Gaser, C. and Schlaug, G., 2003. Brain structures differ between musicians and non-musicians. Journal of Neuroscience, 23(27), pp.9240-9245.

Hanna-Pladdy, B. and MacKay, A., 2011. The relation between instrumental musical activity and cognitive aging. Neuropsychology, 25(3), p.378.

Holland, S., Wilkie, K., Mulholland, P. and Seago, A., 2013. Music interaction: understanding music and human-computer interaction. In Music and human-computer interaction (pp. 1-28). Springer, London.

Holland, S., McPherson, A.P., Mackay, W.E., Wanderley, M.M., Gurevich, M.D., Mudd, T.W., O'Modhrain, S., Wilkie, K.L., Malloch, J.W., Garcia, J. and Johnston, A., 2016, May. Music and HCI. In Proceedings of the 2016 CHI Conference Extended Abstracts on Human Factors in Computing Systems(pp. 3339-3346).

MakeyMakey, http://www.makeymakey.com.

McPherson, A., Morreale, F. and Harrison, J., 2019. Musical instruments for novices: Comparing NIME, HCI and crowdfunding approaches. In New Directions in Music and Human-Computer Interaction (pp. 179-212). Springer, Cham.

Medeiros, R., Calegario, F., Cabral, G. and Ramalho, G., 2014, June. Challenges in designing new interfaces for musical expression. In International Conference of Design, User Experience, and Usability (pp. 643-652). Springer, Cham.

Moggridge, B. and Atkinson, B., 2007. Designing interactions (Vol. 17). Cambridge, MA: MIT press.

Morreale, F., De Angeli, A. and O'Modhrain, S., 2014, June. Musical Interface Design: An Experience-Oriented Framework. In NIME (pp. 467-472).

Paine, G., 2013. New musical instrument design considerations. IEEE MultiMedia, 20(4), pp.76-84.

Saffer, D., 2008. Designing gestural interfaces: touchscreens and interactive devices. " O'Reilly Media, Inc.".

Sharp, H., Preece, J. and Rogers, Y., 2019. Interaction Design-beyond human-computer interaction 5th, 636. isbn: 978-1-119-54725-9.

Tanaka, A., 2019. Embodied Musical Interaction. In New Directions in Music and Human-Computer Interaction (pp. 135-154). Springer, Cham.

Turchet, L., Fischione, C., Essl, G., Keller, D. and Barthet, M., 2018. Internet of musical things: Vision and challenges. IEEE Access, 6, pp.61994-62017.

Turchet, L., 2018. Some reflections on the relation between augmented and smart musical instruments. In Proceedings of the Audio Mostly 2018 on Sound in Immersion and Emotion(pp. 1-7).

Wanderley, M.M. and Orio, N., 2002. Evaluation of input devices for musical expression: Borrowing tools from hci. Computer Music Journal, 26(3), pp.62-76.

Wessel, D. and Wright, M., 2002. Problems and prospects for intimate musical control of computers. Computer music journal, 26(3), pp.11-22. 